\title{
LÉXICO AGRÍCOLA EN EL SIGLO XVII: HERRAMIENTAS DE CORTE
}

\author{
José Ramón Morala Rodríguez \\ Universidad de León \\ jrmorr@unileon.es
}

Resumen: El objetivo del trabajo es revisar la terminología de un campo muy específico del léxico agrícola en el siglo XVII: las herramientas de corte. Es decir, las que podríamos englobar bajo hiperónimos como hoz, hacha, rozón o guadaña. Para ello se utiliza un corpus, el CorLexIn, que se nutre de documentación notarial sobre registros de bienes. El estudio es, por tanto, histórico pero también diatópico, por lo que los datos obtenidos se cruzan con los atlas lingüísticos modernos, en los que esos mismos conceptos suelen aparecer regularmente.

Palabras clave: Léxico agrícola, lexicografía histórica, Siglo de Oro, herramientas, inventarios.

Title: Seventeenth-century agricultural vocabulary: cutting tools.

\begin{abstract}
This study focuses on a review of the terminology related to a very specific field of agricultural vocabulary: cutting tools, that is, those which we could refer to using hypernyms like sickle, axe or scythe. With this purpose, we have used CorLexIn corpus, a corpus based on notarial records that contain mostly inventories of goods. Our work provides a historic perspective, but also diatopical, therefore our results have been collated with the information supplied by modern linguistic atlases, in which these concepts are usually included.
\end{abstract}

Key words: Agricultural Vocabulary, Historical Lexicography, Spanish Golden Age, Tools, Inventories.

\section{INTRODUCCIón}

Nos centramos aquí en analizar con detalle la terminología agrícola usada en el siglo XVII en un campo muy específico, el de las herramientas de corte, es decir, las que se usan en las faenas agrícolas para cortar, podar o segar y que podríamos englobar bajo los hiperónimos hoz, hacha, rozón o guadaña. Los corpus convencionales ofrecen, por lo general, pocas posibilidades de aparición a este tipo de léxico tan concreto y, menos aún, tiene cabida en ellos la variación diatópica que puedan

1 Para la realización de este trabajo se ha contado con la financiación del Ministerio de Ciencia, Innovación y Universidades al proyecto con número de referencia PGC2018-097553-B-I100. 
presentar. Para soslayar este inconveniente, trabajamos sobre un corpus especializado como es el Corpus Léxico de Inventarios (CorLexIn) ${ }^{2}$, constituido a partir de documentación notarial del Siglo de Oro, principalmente el Xvir. En él se incluyen exclusivamente los documentos que registran relaciones de bienes, esto es, inventarios, tasaciones, partijas, cartas de dote, etc. Su mayor interés reside en que, dada la obligación del escribano de registrar todos los bienes de forma minuciosa, este tipo de textos constituye una fuente de datos léxicos de primer orden. Si a eso le añadimos que el corpus cuenta con documentos de la época repartidos por todo el ámbito hispanohablante - aunque aquí me centro únicamente en los españoles-, se comprenderá el interés que tiene para la lexicografía histórica en el ámbito del léxico cotidiano y de la variación diatópica.

Las voces obtenidas por esta vía se comparan, además de con los repertorios léxicos convencionales y con los corpus más generales, con los atlas lingüísticos que, no debería extrañarnos, representan la información equivalente a nuestro corpus con una diferencia de tres siglos: las encuestas directas y los inventarios tienen en común la diversidad geográfica de las fuentes y la recogida de los datos directamente de la declaración de los informantes (Morala, 2012: 421-422). El método nos va a permitir documentar históricamente voces que no figuran en los diccionarios, identificar voces que se dan como generales en los diccionarios, pero solo viven en el ámbito local, o definir isoglosas léxicas, constatando las diferencias que presenten en una y otra época.

\section{HoZ}

Del latín FALCE 'instrumento para segar', hoz es voz general en todas las épocas y con resultados en todos los romances (DECH: s. v. hoz). Como es fácil de suponer, presenta múltiples ocurrencias en el corpus, sin mayor interés que aquellos casos que incluyen diferencias formales o de interés para delimitar el significado. Entre estos, han de señalarse los casos de variación formal en los que, por mantenimiento de la /f-/ o su aspiración, así como por el seseo o el mantenimiento de la /-e/, encontramos voces diferentes al término normativo en castellano:

vna foz (San Román (Piloña), As-1680); una foz y un machado (Caboalles, Le-1644); dos fozes, real y medio (Losazino, Za-1647); vna joz (La Alberca,

2 La consulta del corpus en línea puede hacerse en la web del NDHE. La información relativa al proyecto, con indicación de los archivos visitados, legajos vaciados, un índice de voces estudiadas y la versión en línea de los trabajos publicados, puede verse en $<$ http://corlexin.unileon.es $>$. 
Sa-1665); dos jozes nuebas (Brozas, Cc-1684); dos sachuelos y tres joses de segar (Huelva, 1664); una hoze de podar; una gose de podar (Chiclana de la Frontera, Ca-1670); una hoçe de podar (San Martín de Pusa, To-1532).

Más interesantes son los casos en los que el sustantivo hoz va definido por un verbo o un adjetivo que precisan el sentido, y en consecuencia el valor pecuniario, con el que se usa - algo de vital importancia en una tasación de bienes-, lo que nos permite conocer con más detalle el significado o función de hoz, así como el léxico específico con el que se identifica en los inventarios.

La acepción básica es la de 'hoz para segar' y 'hoz para podar'. De este tipo encontramos abundantes referencias en las que se registra una u otra función con el léxico convencional (segar, podar), lo que, dado que se trataría de objetos distintos aún con un nombre genérico común, muestra la necesidad del escribano de fijar correctamente el sentido de la herramienta que está inventariando. Son ilustrativos los abundantes casos en los que figuran en líneas contiguas de un mismo documento las dos denominaciones: «tres oçes de segar y vna de podar» (Eslava, Na-1631); «vna hoz de podar; tres hoçes de segar» (La Roda, Ab-1643); «dos oçes de segar y vna de podar» (Montefrío, Gr-1661).

No faltan tampoco otras voces más específicas, a veces solo de ámbito local, pero con una referencia similar a las dos anteriores. En el caso de hoz de segar encontramos expresiones como «segar trigo»o «segar pan» — seguramente para diferenciar la siega de hierba - junto a léxico más novedoso como segadera o mesiega. En el primer caso, a partir de segar se ha creado el derivado segadera, que funciona como sustantivo y, con más frecuencia, como adjetivo. En el CorLexIn se documenta en Palencia - también lo hace en el $A L C y L$ (259) en puntos aislados, aunque la RAE lo registra sin marca desde Autoridades (NTLLE: s. v. segadera). En el caso de mesiega o misiega, voz documentada en León ${ }^{3}$ que no figura en el $D L E$, se trata de un derivado de mies, con el sufijo -iego, especialmente activo en el noroeste peninsular (Morala, 2017a: 137-141):

dos oces de segar trigo (Cañedo, S-1608); tres ozes de segar pan (Rupelo, Bu1680); dos ozes segaderas buenas y otra pequeña (Saldaña, Pa-1644); dos oçes segaderas (San Mamés, Pa-1652); quatro foces misiegas; dos fozes mesiegas; dos foces misiegas (Villablino, Le-1646); dos fozes misiegas (Llamas de la Ribera, Le-1586).

\footnotetext{
Aunque no figura en el $D G L A$, Le Men (s. v. misiega) registra joz misiega 'hoz de segar trigo u otra mies' en el área de Sajambre (León).
} 
Por lo que se refiere a la hoz de podar, una herramienta necesariamente distinta y más consistente que la anterior, figura igualmente con diversas denominaciones entre las que destacan, por su frecuencia, podadera y rozadera, que analizamos por separado. Junto a estas, hay otras voces que indican que estamos ante una herramienta usada para desbrozar la maleza del terreno. Así, aparecen la hoz de monte, de rozar árgoma, de atarmar ${ }^{4}$, de segar zarzas, de mata, en muchas ocasiones usadas en un mismo documento por contraposición a las hoces de segar:

vna hoz de podar (Guadalajara, 1625); vna oz de rozar (Cacabelos, 1654); vna hoz de roçar árgoma (Valle de Guriezo, S-1667); tres ozes de roçar árgoma (Torquiendo, S-1669); una foz roçadera; quatro ozes de segar pan (Palacios del Sil, Le-1640); vna oz de monte y quatro misiegas (Cimanes del Tejar, Le1686); una hoz de atarmar y una hoz mesiega (Soto de Valdeón, Le-1647); vna oz de segar zarzas, quatro ozes de segar trigo (Alzaga, SS-1693); una hoz de mata (Cabrojo, S-1631).

La relación de derivados de hoz es relativamente amplia e incluye hocino - el más extendido de todos ellos-, pero también otros de uso más local como hocil, boceta, hocejo y honcejo.

Hocino, con la acepción ${ }^{5}$ de 'instrumento corvo para cortar leña', figura regularmente y sin marca diatópica en la lexicografía académica y, con anterioridad, desde Nebrija, que lo registra como «hocino para segar» (NTLLE: s. v. hocino). En el corpus que manejamos, hocino se registra principalmente en el área centro oriental de Andalucía ${ }^{6}$, área en la que se menciona como herramienta para cortar leña o racimos de uva, pero no fuera de esta zona. Su uso es, sin embargo, más amplio, como puede comprobarse en los atlas, donde, además de en el $A L E A$ (119), figura para podón en puntos de Castilla La Mancha (ALECMan, 236) y para hoz en el castellano norteño $(A L C y L, 259)$ : «vn hocino biexo» (Alcalá la Real, J-1648); «dos ocinillos

4 Este atarmar lo encontramos solo - pero reiterado en varios documentos - en un legajo del Valle de Valdeón, en el ángulo nororiental de León. Es una voz local que no figura en el $D L E$ y tampoco está registrada en Le Men. No obstante, el $D G L A$ (s. v. atarmar) la registra en Cabrales con el sentido de 'podar' y el ALECant (304) da atarmar 'escamujar' en un punto limítrofe con León, ambos testimonios - como el que documentamos nosotros - en el área de confluencia de las tres regiones, en el entorno de Picos de Europa.

5 El término tiene igualmente una acepción topográfica que también aparece ocasionalmente en el corpus: «una bodega en el dicho lugar de Centenera, linde bodega de Alonso el bueno y bodega de Alonso Romero, con vn ocino delantero» (Guadalajara, 1625).

6 Fuera de esta zona solo figura en algún ejemplo aislado de interpretación más dudosa: «vn oçino que sirue de boluedor » (La Solana, CR-1651). 
de cortar vba (Montefrío, Gr-1661); «vn ozino de cortar leña» (Montefrío, Gr1661); «vn hocino» (Tabernas, Al-1655); «un ozino biejo» (Dalías, Al-1651); «vn hoçino nuevo» (Almería, 1659).

Hocil, con esta variante formal, no aparece registrado en la lexicografía general, aunque sí figura en la de ámbito dialectal. Hocil 'herramienta para cortar leña' se registra en el área de León (Le Men: s. v. focín) junto a otros varios derivados de hoz (hocino, fucina, fucin, foucin, etc.). Aunque podría tratarse de un derivado en -ILIS, dada la correspondencia formal y de significado con estas otras formas más frecuentes, quizá hocil se haya originado en focin-hocin, con cambio de la consonante final (Morala, 2017b: 273), aunque ha de tenerse en cuenta la existencia de faucille 'hoz' en francés. En el CorLexIn aparecen dos casos de hocil, ambos en León, pero la nómina de ejemplos es mayor si incluimos las cartas de examen, el registro de las pruebas de aptitud profesional realizadas ante los representantes del gremio correspondiente (Morala, 2008). En este caso, lo habitual en León es que a los aspirantes a herrero se les dé el visto bueno para fabricar una serie de herramientas - que se enumeran con detalle-, entre las que rara vez falta el hocil: «un ocil viejo» (Abadengo de Palacio, Le-1644); «un ozil» (Solanilla, Le-1662); «hacer hachas, machados, acadones, ociles, podaderas» (León, 1643); «haçer machados y açadas y oçes y oçiles» (León, 1643).

El DLE da hocete con el sentido de 'hocino' y marcado como de uso en el ámbito rural de Murcia desde la edición de 1925 (NTLLE: s. v. hocete). Con esta misma terminación lo recogen el ALEA (119) y el ALECMan (374), este con la variante honceta 'herramienta para cortar los racimos', en la zona oriental de la región. En nuestro corpus no encontramos ese derivado pero sí figura, en femenino, un único caso de hoceta, aunque lo hace en un documento del otro extremo de la Península: «vna ozetilla de podar» (Herrera de Valdecañas, $\mathrm{Pa}-1700$ ).

El $D L E$ recoge únicamente la variante incrementada con la nasal, honcejo, y lo refiere, sin más, a hocino. La entrada figura en la lexicografía académica con una definición similar — antes la había registrado Terreros (1787) — desde la edición de 1803 (NTLLE: s. v. honcejo). En nuestro corpus, es más frecuente la forma original (hocejo), ocasionalmente en femenino (hoceja), que la que presenta la nasal (boncejo).

Los ejemplos se concentran todos en León, Palencia ${ }^{7}$ y Cantabria. A estas referencias del corpus pueden añadirse otras similares a lo indicado para hocil: en las cartas de examen para el oficio de herrero escritas en León, figura con frecuencia hocejo entre las herramientas que están autorizados a hacer, una vez superada la prueba:

En el mapa 416 del $A L C y L$ para 'hacha' figura honcejo como respuesta alternativa junto a hacha en un punto del norte de Palencia (Pa-102), mientras que en el 391 'calabozo' hocejo aparece en puntos aislados de Burgos y lo mismo ocurre con honcejo en Segovia y Palencia. 
un honcejo bueno (Castroañe (Cea), Le-1637); un oncexo biejo (Bascones de Ojeda, Pa-1644); tres oçejas y dos arados (Potes, S-1661); vn ozexo y vna cuchilla de hierro (Cifuentes de Rueda, Le-1648); un ozexo y una acha (Herreros de Rueda, Le-1652); un hozejo muy vueno, digo viejo (Mansilla de las Mulas, Le-1638); en açer podaderas, oçejos, achas, açadones y herar bueyes (León, 1643); en azer podaderas, ocejos, achas, azadones y errar bueyes (León, 1643); machados, açuelas, oçejos y oçiles y obra blanca y negra (León, 1643).

\section{PODAR}

Desde el castellano podar, del latín PǓTARE, se han generado diversos derivados que dan nombre a varios de los utensilios propios de esta tarea agrícola. Entre ellos, algunos de uso general como podón o podadera, pero también otros como podejo, - a o podanca, casi desconocidos o poco documentados.

Con el sentido de 'podadera grande y fuerte usada para podar y rozar' $(D L E)$, podón se registra de forma más tardía que podadera, pues solo aparece en la lexicografía española a partir de 1737 en Autoridades (NTLLE: s. v. podón), si bien el CDH ofrece un primer caso en 1387. En cualquier caso, no es voz abundante en el corpus académico, pues suma únicamente 18 ejemplos en el $C D H$. En nuestro corpus se trata de una voz bien representada en el siglo XVII con un buen número de ejemplos y una amplia extensión geográfica: figura en documentos de Palencia, Zamora, Salamanca, Cuenca, Toledo, Toledo, Murcia, Tenerife, etc.: «dos podones» (Ciudad Rodrigo, Sa-1630); «vn podonçillo» (Navahermosa, To-1638); «dos podones» (Cuenca, 1690); «un podón de hierro» (Alburquerque, Ba-1645); «un podón de podar» (Alhama de Murcia, Mu-1648); «vn podón; vna rozadera» (Garachico, Tf-1695).

Además de la forma en masculino ${ }^{8}$, se localiza algún caso del femeninopodona: «un podón en real y medio; una podona en un real» (Figueruela de Arriba, Za1685). Los repertorios léxicos sitúan la voz como habitual en toda el área leonesa, de Asturias a Extremadura (Le Men: s. v. podón, -na) y hay incluso ejemplos en la documentación moderna en Canarias en fuentes del siglo xx (DHCan: s. v. podona), además de ser respuesta frecuente para rozón en el ALEICan (49).

8 En un aprecio de bienes datado en Liébana, en Cantabria, en el que, entre las diversas propiedades, se tasan una larga lista de árboles aislados, especialmente castaños, se usa podón, no con el sentido de herramienta, sino con el de árbol', probablemente por ser árboles que, más que futo, se emplearían para producir leña o madera: «tres castañaricos... otro castañar... más un podón azia la sierra de Cabañes... más otro que llaman el castañal berde... otro podón junto al dicho... vn nogal, el mayor, junto a la yglesia» (Pendes, S-1657). 
Podadera, 'herramienta acerada, con corte curvo y mango de madera o hierro, que se usa para podar', es el resultado de la abreviación de foz podadera (DECH: s. v.podar) y se registra como sustantivo en la lexicografía hispánica desde Nebrija, sin mayor variación que algunas acepciones más descriptivas (NTLLE: s. v. podadera). Es voz de uso general en todas las épocas y zonas y, en nuestro corpus, se usa como sustantivo con ejemplos repartidos por toda el área de estudio, a veces con especificaciones interesantes («de viñas», «de bocado», «de yerro») sobre su forma o el uso que se le da: «una podadera nueba y otra bieja» (Toro, Za-1607); «vna podadera de viñas» (Navahermosa, To-1638); «huna podadera de yerro biexa» (Soria, 1637); «una podadera de bocado» (Logrosán, Cc-1675); «vna podadera para podar la viña» (Almería, 1659).

Entre las variantes de interés, puede destacarse el hecho de que perviva algún ejemplo de su uso como adjetivo (hoz podadera) - como el citado para Palenciay la presencia ocasional en los inventarios de una forma en masculino (podadero), localizada en el área occidental que, sin embargo, los repertorios léxicos de la zona ( $D G L A$, Le Men) no nos confirman actualmente y que podrían explicarse por el uso adjetivo junto a un sustantivo masculino (cuchillo podadero): «una hoz podadera» (San Mamés, Pa-1652); «dos cochillos podaderos» (Toranzo, S-1622); «un podadero» (Villarino de Manzanas, Za-1683).

El término podanca no lo encuentro ni en la lexicografía (NTLLE) ni en los corpus académicos, ya sean históricos o actuales. El contexto en el que se localiza el único ejemplo que registra el CorLexIn ofrece escasas dudas sobre su interpretación, pues la denominada podanca se cita junto a otras herramientas como el corvillo, la podadera o el calabozo. Dada la costumbre de los escribanos de organizar los inventarios por áreas temáticas, estaríamos ante un derivado del que no tenemos otras referencias, pero que necesariamente hemos de interpretar como un tipo de podón o podadera: «dos corvillos y vna podanca; tres podaderas; vn calahoço» (Albalá, Cc-1661).

En lo tocante a podejo, de nuevo nos encontramos con un localismo no registrado en la lexicografía académica, si bien en este caso contamos con más de un ejemplo en el corpus y, además, con alguna referencia actual: J. Le Men (s. v.podeja) incluye esta voz, ahora en femenino y con el sentido de 'instrumento de hierro con un mango de madera parecido a la hoz y que sirve para podar'. La localiza en el valle del Esla, al oriente de León y no lejos de la ubicación en Sahagún que ofrece el CorLexIn. Un sentido similar ${ }^{9}$ tendría seguramente en los textos de Sahagún de

9 Lo único extraño es la referencia, en uno de los ejemplos, a majar el lino y no a podar. En cualquier caso, la familia léxica de podar tiene en este repertorio léxico de León otras varias formas poco documentadas (podaja, poderón, podeyón, podina) que dan fe de sus posibilidades derivativas. 
comienzos del siglo XVI: «un podexo, y una çoleta, y una oz de segar» (Sahagún, Le-1601); «vn podexo pequeño» (Sahagún, Le-1608); «dos podaderas demediadas; dos podexos de maxar lino» (Sahagún, Le-1608).

\section{3. $\operatorname{ROZAR}$}

De modo similar a lo que hemos visto para podar, el verbo rozar es igualmente el punto de partida para la denominación de varias herramientas. La más frecuente es rozadera, pero también hay algunas otras voces de uso más local, como rozón, ambas repitiendo el esquema de podar / podadera / podón visto arriba. Del latín RŬPTIARE, el castellano rozar tiene como significado primario el de limpiar las tierras de las matas y hierbas inútiles antes de labrarlas', aunque inicialmente tuvo el sentido de 'roturar, arar un campo por primera vez' (DECH: s. v. rozar $)^{10}$.

Rozón aparece tardíamente en los registros lexicográficos. No figura hasta la edición del diccionario académico de 1884 , donde entra por primera vez con la acepción de 'especie de guadaña tosca, corta, gruesa y ancha, que, sujeta á un mango largo, sirve para rozar árgoma, zarzas, etc.', que se mantiene hasta la actualidad, sin marca diatópica alguna. Los datos del $C D H$ no son muy diferentes, pues se documenta solo a partir de finales del siglo XIX, en textos mayoritariamente relacionados con Cantabria, lo que confirman los atlas, con un uso mayoritario de rozón 'instrumento para rozar' (ALECant, 127). Esta misma localización regional ${ }^{11}$, pero ahora en el siglo XVII, tienen los ejemplos aportados por el CorLexIn, todos ellos procedentes de la documentación del archivo cántabro: «una acha y un roçón» (Tanos, S-1672); «vn roçón y una hoz» (Cudón, S-1672); «vn roçón viejo» (Torrelavega, S-1673). El uso de rozar en Cantabria parece especialmente vivo en los documentos del corpus. En un par de ocasiones se cita «una hoz para rozar árgoma» (Guriezo, S-1669 y 1676) y ha de añadirse una forma compuesta de claro uso local, rozaescajos, es decir, un tipo de hoz que serviría para desbrozar de escajos las fincas ${ }^{12}$ : «un roçaescajos nuebo» (Cañedo, S-1615).

10 El verbo lo encontramos en el corpus en la expresión hoz de rozar, pero también en otras como «un açadón de roçar» (Moratalla, Mu-1628) o en los topónimos formados sobre roza 'terreno roturado o desbrozado'.

11 Aunque carezcamos de ejemplos aquí, rozón es también una voz bien registrada en Asturias, con testimonio escrito al menos desde 1788 (DGLA: s. v. rozón).

12 Voz de uso local en Cantabria para aulaga $(D L E)$, que se extiende también por Asturias (DGLA: s. v. escayu) y el noroeste de León (Le Men: s. v. escayadal), en estos dos casos principalmente con el sentido de 'espino'. 
El segundo derivado de rozar, rozadera, se documenta aún más tardíamente en la lexicografía académica: solo lo hace a partir de la edición de 1925 del $D R A E$, inicialmente remitiendo a 'rozón' y, desde 1970, como 'especie de guadaña para quitar matas y hierbas inútiles', siempre sin marca alguna de carácter diatópico (NTLLE: s. v. rozadera). La consulta en el $C D H$ ofrece resultados aún más escasos, pues solo devuelve dos resultados que poco tienen que ver con el que aquí nos interesa.

Sin embargo, el término rozadera - ya sea como adjetivo, ya como sustantivo- parece estar ya bien asentado en el siglo XvII y, lo que es más interesante, se documenta ocupando un área mucho más extensa que la de rozón. Bien es verdad que es mucho más abundante en la documentación del noroeste leonés - Laciana y Alto Sil, donde aparece casi regularmente como foz rozadera o rozadeira en la mayoría de los inventarios-, pero asimismo ocupa un territorio más amplio, que alcanza a Soria, Ávila, Zaragoza, Badajoz o Tenerife:

una foz roçadeyra y quatro de segar pan (Villablino, Le-1647); una foz rozadera (Villablino, Le-1646); una hoz roçadera (Matalvilla, Le-1641); una podadera roçadera (Valdelaguna, Áv-1651); una azada roçadera ( Noviercas, So-1653); dos roçaderas (Fregenal dela Sierra, Ba-1666); azada escabadera y rozadera (Maluenda, Z-1647); vna rozadera; tres podones (Garachico, Tf-(1695).

\section{AZUELA}

Azuela, con el sentido de 'herramienta de carpintero para desbastar', procede del diminutivo hispanolatino de ASCIA 'azuela, hacha', AsciŏLA (DECH: s. v. azuela) y es voz de uso general en la lexicografía hispánica desde Nebrija, con la variante açuela en los diccionarios más antiguos (NTLLE: s. vv. azuela y açuela).

Como término de uso antiguo y general que es, en nuestro corpus se registra de forma abundante por todo el ámbito de estudio. Las únicas variantes formales que localizamos son una forma en masculino (azuelo) que, por el contexto, ha de tener un sentido diferente, varios casos de zuela en Zamora y León —con aféresis de la /a-/ inicial- y un caso de ajuela, con el resultado palatal del aragonés ${ }^{13}$ para el grupo /skj/: «un açuelo de escardar» (Santa María la Real de Nieva, Sg-1643); «vn ajuela con su martillo» (Eslava, Na-1631); «vna zuela ancha y otra angosta» (Llamas de la Ribera, Le-1586); «un zadón y una zuela; una zada» (Mahíde, Za-1664).

13 En otros documentos se usa el castellano azuela: «dos azuelas, dos segures» (Cortes, $\mathrm{Na}-1645)$. 
Quizá lo más interesante en el uso de azuela sea la frecuencia con la que se especifica su uso o su forma, dando lugar a un variado muestrario léxico, especialmente cuando se identifica con un verbo o un adjetivo el uso concreto para el que sirve un determinado modelo de azuela. Así, junto a verbos como dolar, labrar, aperar ${ }^{14}$, botanar ${ }^{15}$, escardar o acepillar, vemos adjetivos como desmontadera (de desmontar 'cortar la maleza, rozar'), descepadera (de descepar 'arrancar de raíz la cepa'), dornillera (para hacer dornillos) ${ }^{16}$, cambera (para labrar cambas 'cama, pieza de la rueda o el arado) o abarquera (para fabricar abarcas 'zuecos'), en la línea de los más extendidos podadera o rozadera.

una açuela de mano de dolar (San Mamés, Pa-1652); una azuela de labrar madera (Casarejos, So-1644); una azuela de aperar (Moratalla, Mu-1632); dos açuelas de botanar (Casarejos, So-1648); una azuela de escardar (Villaescusa, Za-1645); una açuela de açepillar (El Espinar, Sg-1657); vna hazuela desmontadera (Cebreros, Áv-1651); vna haçuela desçepadera (Cebreros, Áv-1652); una açuela de dos bocas, dornillera (Cebreros, Áv-1654); vna azuela de cabestro; otra cambera; otra abarquera quebrada (Otero de Guardo, Pa-1654).

Algo similar ocurre cuando se describen el aspecto o los componentes específicos de esta herramienta. Figuran por esta vía en la documentación azuelas de peto I de peta, de cabestro, de mano, de corte, de martillo, de cotillo o bien ancha, estrecha $\mathrm{o}$ angosta:

vna haçuela de corte (Cuenca, 1651); una azuela con su cabestro (Tábara, Za1688); vna azuela de peto (Saldaña, Pa-1644); una açuela de mano, de martillo (Cebreros, Av-1651); una açuela de cotillo (Aguilafuente, Sg-1623); vna azuela ancha y otra angosta (Herreros de Rueda, Le-1652); dos açuelas, la una ancha y la otra arisca (Guijo de Granadilla, Cc-1671); dos azuelas, la una de peto y otra de cabestro (Santas Martas, Le-1625); una azuela peta (San Miguel, Le-1647); dos açuelas, una pata y otra peta (Villablino, Le-1648).

\footnotetext{
14 Aunque hoy figura como de uso general en el DLE, en Autoridades, donde se registra por primera vez, aperar cuenta con una restricción geográfica para La Mancha y Murcia, localizándose el ejemplo en esta última zona (NTLLE: s. v. aperar).

15 El $D L E$ da botana 'pequeño tarugo de madera que se pone en las cubas para que no se salga el vino', pero no registra el verbo botanar, que tampoco localizo en el $C D H$. nos, Áv-1658).

16 En la misma provincia, encontramos «una achuela de guvia para açer dornillos» (Pegueri-
} 
Con una sola ocurrencia en el CorLexIn encontramos el derivado de azuela, azolijo, en un documento de Ávila. El contexto se refiere, desde luego, a una herramienta de corte. Sin embargo, azolijo no se registra ni en el NTLLE ni en el CDH: «vn calaboço, en seis reales; vna hacha de cortar, en cinco reales; vn açolijo pequeño, en tres reales» (Candeleda, Áv-1648). Seguramente se trata de una voz local, pues en internet es posible localizar usos de esta palabra en textos radicados en el Valle del Tiétar, la misma área en la que se documenta en el siglo XVII. En uno de esos textos, un vocabulario de Guisando ${ }^{17}$ registra la entrada azolijo con el significado de 'instrumento de hierro que consta de un hacha y una azada pequeña'.

\section{CALABOzO}

El DLE registra calabozo - junto con la variante calagozo - con el sentido único y carente de marcas de 'instrumento de hoja acerada, ancha y fuerte, para podar y rozar árboles y matas'. Ambos están recogidos desde Autoridades, que prefiere calagozo, si bien Covarrubias había registrado ya únicamente la variante calabozo. Con /b/ figura también en Nebrija (calaboço) y en todos los diccionarios preacadémicos. En la lexicografía académica, hasta la edición de 1899 se añade la marca diatópica de que se usa en «Extremadura, Andalucía y otras partes», que desaparece en la de 1914 (NTLLE: s. vv. calabozo y calagozo).

El $D E C H$, que lo define como 'especie de podadera', duda sobre su origen: «probablemente compuesto de calar 'penetrar, atravesar' y leonés boza 'matorral', 'roza, rompido', de origen prerromano», apoyándose en las formas occidentales boza, bouza; aunque, con este origen, les extraña la forma en /-o/. Por otra parte, J. Corominas y J. A. Pascual la consideran una «palabra principalmente leonesa» (DECH: s. v. calabozo II).

La distribución geográfica en nuestros documentos del siglo XVII no se corresponde, sin embargo, con lo que esperaríamos para una voz considerada propiamente leonesa. El término, que es muy abundante en Zamora, aparece también en Salamanca — con algún ejemplo hacia la zona cercana de Ávila—, pero, al norte del dominio, carecemos de ejemplos en Asturias y solo se documentan casos aislados en el sur de la provincia de León ${ }^{18}$, aunque no en el resto de la provincia. En esta área, si

17 Concretamente aparece en el número 85 (junio de 2017) de El periódico del Tiétar. Hay igualmente alguna referencia en la zona de Toledo, con la que linda esta comarca abulense. Así, se documenta el azolijo 'herramienta parecida a la azuela pero con dos cortes' en la fabricación artesanal de rabeles en Las Ventas de San Julián (González Casarrubios, 1978: 25). El ALECMan (128) también registra en un punto del norte de Toledo la variante azolejo 'escardillo'.

18 En el caso de León, los ejemplos escasean y únicamente aparecen algunas referencias al sur 
bien se registran algunas variantes como calabuzo ${ }^{19}$ o el académico calagozo, la más frecuente es calabozo:

una podadera y un calabozo (La Bañeza, Le-1641); un calabozo y un machado (Tábara, Za-1687); un azadón y un calabozo (Riofrío de Aliste, Za-1688); un calavoço de yerro (Ciudad Rodrigo, Sa-1630); vn calaboço (Candeleda, Áv1648); un calabuzo desbocado (Alija del Infantado, Le-1644); un calabuzo de yerro (Coomonte de la Vega, Za-1650); vn calagoço (Villarino de Manzanas, Za-1683); un machado; un calagozo (Muga de Alba, Za-1647).

Más al sur, el término es de uso frecuente en la documentación extremeña - a la que se uniría un ejemplo de Toledo en un punto cercano a Cáceres-, lo que corroboraría la ascendencia leonesa de calabozo, de la que habla el $D E C H$, y, en menor medida, un foco de ejemplos en documentación andaluza ${ }^{20}$ en Cádiz y Málaga:

vn calahozo (Navahermosa, To-1638); una segureja; un calahoço (Logrosán, Cc-1672); un calahoço (Albalá, Cc-1661); un calagoço y un segurón, (Guijo de Granadilla, Cc-1664); un calahoz (Logrosán, Cc-1678); un calahoze; otro calahoze (Logrosán, Cc-1688); vna sigureja y un calahoze (Logrosán, Cc1668); vna hacha y un calaboso (Arcos de la Frontera, Ca-1666); vn calabosso (Álora, Ma-1661); una hacha; dos calabosos (Puebla de Peñarubia, Teba, Ma1699).

Al margen de la grafía seseante de Málaga y Cádiz, calaboso, es muy interesante la variante mayoritaria que se registra en Cáceres, con pérdida de la oclusiva intervocálica /b/ (calabozo), que probablemente haya que considerar como un paso intermedio hacia el calagozo, siempre minoritario, pero que la RAE da como entrada de referencia. Igualmente, entre las variantes formales documentadas en este bloque de ejemplos, ha de reseñarse la aféresis de la vocal final (calahoz) y la posterior epéntesis de /-e/ (calaboce) que presentan algunas ocurrencias del corpus.

de la provincia, en el límite con Zamora. De hecho, en el CorLexIn no hay ocurrencias para León y estos ejemplos los he tomado de un trabajo específico sobre el área leonesa cercana a Benavente (Gómez Ferrero, 2015: 259).

19 Las variantes con /u/, calabuzo, resultan especialmente extrañas pues el cierre de /o/ en $/ \mathrm{u} /$ afecta a la vocal tónica, algo que no es habitual. Todos los casos están localizados en una zona muy concreta, en el límite entre León y Zamora (Gómez Ferrero, 2015: 259).

20 Recordemos que esta es la distribución (Extremadura y Andalucía) a la que aluden algunas ediciones antiguas del diccionario académico. 
Finalmente, las isoglosas que dibuja nuestro corpus para calabozo, más que una voz marcadamente leonesa - no parece que se haya usado al norte del dominio-, le dan un carácter occidental, en el sentido amplio de la palabra que definió M. Ariza (1995), usada en un espacio que va, de norte a sur, desde el límite de León con Zamora hasta Cádiz, con una tímida expansión hacia áreas limítrofes del dominio histórico del castellano (Ávila, Toledo) y, algo más alejado, hacia Málaga. Es posible, sin embargo, que el término haya logrado posteriormente expandirse, pues en los atlas, además de en el área centro-occidental andaluza $(A L E A, 119)$ y en las provincias de Zamora y Salamanca $(A L C y L, 391)$, lo encontramos extendido por buena parte de León, así como en puntos de Burgos, Soria, Ávila y La Rioja $(A L C y L, 391$; ALEANR, 337).

\section{CORVO, CORVILLO}

El sustantivo corvo con el sentido de 'garfio' se registra en el NTLLE (s. v. corvo) desde Autoridades. En ediciones modernas, desde el diccionario manual de 1983 se le añade la acepción de 'machete curvo utilizado en la labranza' con la marca de «en algunos países de América», marca que desaparece en la edición de 2001, manteniendo la acepción indicada.

Más frecuente parece ser el diminutivo lexicalizado corvillo, que el $D L E$ registra únicamente en la expresión «miércoles corvillo»y, solo en algunas ediciones, figura corvillo — corbillo en las más modernas - con el sentido de 'espuerta de mimbres' y la marca de Aragón. Con el sentido de recipiente, figura en nuestro corpus algún ejemplo de corbillón, especialmente en el área de Murcia y Albacete. No obstante, hay también ejemplos de corvillo en contextos en los que parece claro que se refiere a algún tipo de herramienta de corte y que, con este sentido, remitiría sin duda a corvo, que el $D L E$ registra en una de sus acepciones como 'machete curvo utilizado en la labranza'. El $D E C H$ (s. v. corvo) reúne varios ejemplos de corvo y corvillo en castellano y en leonés con el sentido que aquí utilizamos y que se ve en casos como estos: «dos corbillos podaderos» (Toranzo, S-1622); «dos oçes corbillas» (Arroyuelos, S-1658); «un corbillo de hierro» (Logrosán, Cc-1675); «dos corvillos y vna podanca» (Albalá, Cc-1661); «tres podaderas y un corbillo de hierro; otro corbillo y dos jozes nuebas» (Brozas, Cc-1684).

Los dos primeros ejemplos corresponden a Cantabria y corvillo se utiliza bien como sustantivo («corbillos podaderos»), bien como adjetivo («oçes corbillas»), pero en ambos casos en un contexto suficientemente claro. Algo similar ocurre en los ejemplos extremeños, de los que tres aparecen junto a otras herramientas de cor- 
te. Aunque no esté registrado en la lexicografía académica, los atlas lingüísticos nos permiten rastrear aún la existencia de esta voz, si bien solo en puntos aislados: en CLEx (174) hay un punto al norte de Cáceres (Cc-100) en el que se da corvillo para «herramienta para cortar los racimos». Algo similar ocurre en el ALECMan (374) en el que, para idéntica pregunta, hay una respuesta (To-203) que registra esta misma voz ${ }^{21}$. También puede hablarse de continuidad en Cantabria, donde el ALECant ofrece para 'calabozo' la respuesta corvillo, en varios puntos del extremo oriental del área estudiada. Casos similares se detectan en Salamanca en el $A L C y L$ (391) y en Andalucía (ALEA, 119).

\section{OTROS TÉRMINOS}

Relacionados de una u otra forma con el concepto de 'hoz de podar', se localizan en el CorLexIn algunos otros términos cercanos, como podría ser castradera 'instrumento de hierro que sirve para castrar las colmenas', que en cierto modo recuerda la forma de la hoz y que probablemente se ha originado de forma similar a lo visto para rozadera o podadera. El $C D H$ tan solo devuelve un ejemplo usado en contexto - el primero que documenta es el diccionario de Nebrija-, por lo que pueden ser de interés lo casos de uso que proporcionan los inventarios: «una castradera » (Tábara, Za-1688); «una castradera de garavato y una podadera » (Cebreros, Áv-1653); «una crastadera» (Guijo de Granadilla, Cc-1669); «una castradera de fierro» (Adeje, Tf-1695).

Finalmente, un documento de Tenerife nos proporciona un par de voces que no localizamos en más ocasiones en el corpus. Se trata de gabite y márcola, que aparecen en un contexto bastante obvio para determinar su significado: «una márcula con que se podan los olibos; una sigura y gabite» (Adeje, Tf-1695).

De ellos, gabite no aparece en el $D L E$ y no hemos localizado otra información, ni siquiera en el DHECan o en el DEC. Por su parte, el DLE sitúa márcola en Andalucía occidental y lo define como «asta de unos dos metros y medio de largo, que lleva en la punta un hierro a manera de formón» para podar los olivos, definición que, en esencia, mantiene la que ya presentaba en la edición de 1803, cuando se registra por primera vez en la lexicografía académica. Con anterioridad lo hace en otros varios diccionarios, el primero de ellos el de Casas (1570), que lo traduce al latín por ronca 'podón' (NTLLE: s. v. márcola).

21 Además, el ALECMan (177) recoge en dos puntos de Cuenca corbella para 'hoz' y en otro de Albacete corbilla. En estos casos, estará relacionado con el valenciano corbella 'hoz' (DECH: s. $v$. corvo). 
La documentación histórica de márcola es escasa. No figura en el $D E C$, ni se documenta en el DHECan, mientras que el $C D H$ devuelve únicamente un ejemplo ${ }^{22}$, ya del siglo XIX. Más abundantes son las referencias para Andalucía - tanto $\operatorname{del} A L E A$ (119) como de otras fuentes-, donde se registran márcola - en menor medida márcula - y el verbo marcolar 'podar' (Alvar Ezquerra, 2000: 508).

\section{HACHA}

Hacha, tomado del francés hache (DECH: s. v. hacha II), se registra de forma regular en inventarios repartidos por toda la Península a lo largo del periodo estudiado. Sin variantes formales reseñables salvo algún caso con aspiración inicial ${ }^{23}$, únicamente cabe destacar la frecuencia con la que el sustantivo se especifica con expresiones como «de cortar leña », «de cortar», «de partir leña », «de monte», «de enarcar». Lo que el escribano está poniendo de manifiesto es la necesidad de evitar confusiones - téngase en cuenta la necesidad notarial de identificar los bienes sin asomo de duda - con la voz homónima hacha 'vela grande de cera', habitual igualmente en este tipo de registros de bienes (Morala 2019: 392-393): «tres achas de enarcar» (Santas Martas, Le-1625); «un acha de peto» (Casarejos, So-1644); «una hacha de monte» (Aguilafuentte, Sg-1623); «dos achas pequeñas de partir leña» (Madrid, 1657); «una hacha de cortar madera» (Montefrío, Gr-1662); «una hacha de yerro» (Dalías, Al-1651).

Entre los derivados que se pueden localizar en el corpus que nos sirve de referencia no figuran ejemplos de hacheta y tan solo aparece un caso de hachón, en el que, como arriba, se especifica con el consabido «partir leña » para evitar la homonimia ${ }^{24}:$ «vn achón de partir leña» (Illescas, To-1626). Fuera de este, los derivados de hacha que están bien representados son hacho y hachuela.

El DLE registra hacho 'hacha pequeña de cortar', pero lo hace solo desde la edi-

22 «Cuando la patria peligra todos somos soldados, unos con fusil, bayoneta y sable, y otros con chuzos, márcolas, garrotes ó piedras» (Fray Francisco Alvarado, 1811).

23 En documentos meridionales se localizan algunos ejemplos en los que hacha figura con la grafía «j-», como muestra gráfica de la aspiración de la inicial: «dos jachas de partir leña (Bollullos Par del Condado, H-1657); «vna jacha y un josino maltratados» (Benamargosa, Ma-1672). Lógicamente, incluso en los documentos que muestran tendencia a marcar la aspiración con dicha grafía, aparece también casos con la grafía convencional; «dos hachas de jierro» (Teba, Ma-1699). La aspiración es la norma general en Andalucía, salvo en el oriente de la región ( $A L E A, 716)$.

24 El DLE registra hachón únicamente con el sentido 'antorcha, luminaria' tomado de hacha 'vela grande de cera'; pero ejemplos como el referido nos indican que también existió en castellano el derivado homónimo de hacha 'herramienta para cortar'. Del mismo modo, el término está atestiguado por los atlas lingüísticos en puntos aislados como Teruel $(A L E A N R, 339)$ o Málaga $(A L E A, 716)$. 
ción de 1970. Con anterioridad, tanto los diccionarios académicos como los no académicos registran hacho sistemáticamente con el sentido de 'antorcha'. Únicamente Minsheu (1617) nos da cuenta del uso de hacho con el sentido que aquí nos interesa, definiéndolo como «hacho de leña, fascis coculorum» (NTLLE: s. v. hacho). Los atlas lingüísticos registran hacho como alternativa a hacha en puntos del norte de Castilla y León $(A L C y l, 391$ y 416), en Cantabria (ALECant, 1039) y está muy extendido en Asturias (DGLA: s. v. hachu).

El uso histórico de hacho con el sentido de herramienta de corte cuenta con abundantes ejemplos en el CorLexIn extendidos por buena parte de la Península - con mayor presencia en la mitad norte-, en los que ocasionalmente se indica que sirve para «partir leña». Con frecuencia figura relacionado en series con otras herramientas similares (azuela, hoz) y no faltan inventarios en los que se inventaría junto a hacha, lo que indicaría que el significado de ambas voces era diferente: «un acho, una foz» (Premoño, As-1668); «un acho de partir leña» (Castroañe, Le-1637); «un acho bueno y una acha pequeña» (Valderrábano de Valdavia, Pa1642); «quatro hozes de segar y dos achos» (Carazo, Bu-1680); «una acha buena $\mathrm{y}$ un acho» (Vadillo, So-1650.

Por lo que toca a hachuela, figura en el $D L E$ únicamente con el sentido de 'hacha de abordaje', aunque desde Autoridades ('hacha pequeña de partir leña') hasta la edición de 1984 figuraba también como 'diminutivo de hacha de cortar' (NTLLE: s. v. hachuela). Es posible que en la documentación del corpus se use simplemente como diminutivo, pero en varios de los ejemplos parece utilizarse más bien como una forma lexicalizada ${ }^{25}$, especialmente cuando se registra junto a la forma simple hacha. Los atlas lingüísticos dan hachuela como forma alternativa a hacha en amplias áreas de Aragón (ALEANR, 339) y en puntos de Andalucía (ALEA, 601).

La variante está ampliamente extendida por la Península en el siglo XVII y, cuando se detalla, se hace como «de hierro», «de mano» o «de partir carne». Cabe destacar la grafía jachuela, como muestra de aspiración en un documento malagueño:

vna acha grande; una achuela de mano (Arroyuelos, S-1658); una oz de segar y una achuela (Sahagún, Le-1601); tres achuelas de partir carne (Cuenca, 1631); tres achuelas de mano (Guadalajara, 1625); una achuela pequeña (Noviercas So-1653); vn hocino biexo; dos hachuelas (Alcalá la Real, J-1648); vna ha-

25 Si realmente se usara como diminutivo, sería mucho más lógico que hacha apareciera también incrementado por otros sufijos de uso más frecuente en la época y con abundante presencia en el corpus. Sin embargo, no se localizan ejemplos de hachilla, hachica, hachita, hachina o similares, lo que refuerza la idea de que el derivado en -uela está lexicalizado. 
chuela con su peto (Almería, 1659); vna jachuela de hierro (Cútar, Ma-1672); una achuela y dos açadicas (Caravaca, Mu-1654).

\section{MACHADO}

En el DLE machado figura con el significado de 'hacha para cortar madera', sin marca alguna. No obstante, cuando aparece en Autoridades $(1734)^{26}$, se indica que es «voz provincial de Galicia», referencia geográfica que desaparece ya en la edición de 1803 . Originalmente es participio de machar 'machacar', que a su vez procede de macho 'mazo grande', variante de mazo, probablemente de origen mozárabe; las variantes que citan J. Corominas y J. A. Pascual con el sentido de 'hacha' se localizan en Zamora o Extremadura, además de en portugués (DECH: s. v. mazo II).

La distribución que ofrece nuestro corpus permite localizar el término en abundantes inventarios, pero todos ellos proceden del área occidental, concretamente de las relaciones de bienes datadas en León y en Zamora ${ }^{27}$. El sentido de 'herramienta de corte' es evidente por los contextos en los que se cita, con frecuencia en enumeraciones junto a hoz, calabozo o azuela, aunque en alguna ocasión también debe hacer referencia («un machado de carne») a un útil de cocina. Entre las variantes, únicamente cabe citar que, aunque la forma más general es en masculino, se apunta una forma en femenino (machada) que, dado que aparece en contextos junto al masculino, ha de representar un sentido distinto, tal vez diferenciados por uso o por tamaño:

una acha; una machada, un machado de carne (Matalavilla, Le-1636); vn machado pequeño sin mango (Astorga, Le-1692); un machado de peto; una machada (Abadengo de Palacio, Le-1644); vn machado biexo (Valderas, Le1647); una machada (Mansilla de las Mulas, Le-1638); vn machado de cueta (San Román de los Caballeros, Le-1585); un machado, el mejor (Villamor de

26 Con anterioridad al primer diccionario académico, el NTLLE (s. v. machado) solo lo detecta en Stevens (1706): «machádo: a hatchet».

27 Esta misma distribución presenta en el $A L C y L$ (416), donde machado, a veces en alternancia con macheta, ocupa la mayoría de las respuestas para 'hacha' en León y en Zamora. Por lo que toca a macheta, figura también en un buen número de vocabularios del dominio leonés (Le Men: s. $v$. macheta) y los diccionarios académicos registran como voz del ámbito dominio leonés (NTLLE: s. $v$. macheta), pero no aparece en nuestro corpus del XVII, aunque lo hace en documentación más tardía (Gómez Ferrero, 2015: 307), por lo que seguramente es una voz de expansión más moderna. Sí hay registro documental de machete, si bien con mayor incidencia en la documentación americana que aquí no incluyo: «vn machete con su bayna» (Alcalá la Real, J-1648); «un machete pequeño» (Bercial de Zapardiel, Áv-1650). 
Cadozos, Za-1643); un machado (Riofrío de Aliste, Za-1688); un calabozo y un machado (Tábara, Za-1687); vn machado de dos cortes; vn machado de un corte (Abejera, Za- 1689); vn machado (Losacio, Za-1670); vn machado (Mahíde, Za-1664).

Curiosamente, si bien la distribución geográfica de machar 'machacar' es bastante más amplia en nuestro corpus - generalmente referido al lino machado - las referencias al machado 'herramienta' se ciñen únicamente al área leonesa, en línea con lo que muestra la dialectología actual, que circunscribe su uso a todo el occidente peninsular y donde, con diferentes significados, se registran tanto machado como el menos frecuente machada (Le Men: s. v. machado). Para las partes del machado se cita el peto, con el sentido que tiene en castellano, pero también la cueta, voz que no registra el $D L E$, pero que en asturleonés significa 'parte opuesta al filo de cualquier herramienta' (DGLA: s. v. cueta; Le Men: s. v. cueta).

\section{SEGUR}

Resultado del latín SECŪRE 'hacha', segur, con la acepción principal de 'hacha para cortar', es voz antigua en castellano, reflejada en los repertorios lexicográficos desde Nebrija. En la mayoría de ellos se consignan igualmente los derivados segureja y segurón, con pequeños matices de significado sobre el sentido de la forma base (NTLLE: s. vv. segur, segureja, segurón). La voz parece proceder de un derivado del latín SECARE 'segar', aunque no es del todo claro (DECH: s. v. segar) e históricamente se documentan también las variantes formales asegur y segura (NTLLE: s. v. asegur, segura).

Tanto la forma simple como los derivados que se crean sobre esta raíz tienen una apreciable presencia en el CorLexIn, si bien su distribución geográfica no es homogénea y presenta algunas particularidades de interés. Una de las áreas en las que aparecen ejemplos de segur, pero no de sus derivados, es la navarroaragonesa, con ejemplos en Teruel y Navarra en los que parece que se asocia más a 'azada' que a 'hacha', algo que es evidente en el primero de los casos: «vna açada segur buena» (Villalba Baja, Te-1641); «vna açada y vna segur andada» (Eslava, Na-1631); «dos azuelas, dos segures» (Cortes, Na-1645).

El otro núcleo que podemos identificar, aquí con abundante presencia de las formas sufijadas y con una mayor variedad formal, es el formado principalmente por los documentos procedentes de los archivos de Ávila - en este caso solo derivados- y Cáceres, así como algunos otros menos frecuentes de León, Zamora o 
Segovia. Para la forma simple, la variante más frecuente incluye una /-a/ (segura), inducida seguramente por una adecuación formal al femenino. Ha de añadirse igualmente algún caso de cierre de /e/ en /i/ en la sílaba inicial (sigura) ${ }^{28}:$ «dos hachas y una segur» (Soto de Valdeón, Le-1648); «vna segur» (Tábara, Za-1688); «una segura $\gg$ (Figueruela de Arriba, Za-1685); «una oz, dos siguras» (Segovia, 1671); «un calagoço y una segura» (Guijo de Granadilla, Cc-1664).

Por su parte, el derivado segurón presenta casos únicamente en Ávila ${ }^{29}$ y Cáceres, entre los que hay también alguno con cierre de la vocal inicial (sigurón) y con algunos ejemplos en los que se diferencia de hacha («una hacha, digo sigurón» [Navatejares, Av-1652]), de segur («una segura y un segurón» [Guijo de Granadilla, Cc-1665]) o de segureja («vn sigurón y una sigureja» [Albalá, Cc-1661]): «un segurón y un legón» (Valdelaguna, Áv-1651); «un segurón» (El Barco de Ávila, Av-1653); «un calagoço y un segurón» (Guijo de Granadilla, Cc-1664).

Finalmente, segureja cuenta también con ejemplos en documentos con la misma procedencia que el anterior y, como antes, encontramos de nuevo la variante con cierre de la inicial (sigureja): «una segureja pequeña» (Barco de Ávila, Áv-1652); «vna sigureja y un calahoze» (Logrosán, Cc-1668); «una sigureja» (Cáceres, 1649); «una segureja sin astil; una segureja torreznera» (Logrosán, Cc-1677).

Pese a que el término no se registra con este sentido en los diccionarios, la gama de derivados se completa con dos casos de segurilla — uno en cada una de las dos provincias que venimos citando- para los que el contexto no permite saber si se trata de una forma lexicalizada o simplemente de un diminutivo, si bien la dialectología actual lo registra en los atlas lingüísticos como sustantivo independiente: «una segurilla y oxo de otra» (Santiago del Collado, Áv-1651); «una segurilla torreznera $\gg^{30}$ (Logrosán, Cc-1674).

Para cerrar la relación de formas de segur localizadas en el corpus, ha de añadirse un ejemplo de sigura localizado en Tenerife y que, por tanto, presenta una clara

28 La variación entre vocales palatales en posición inicial átona es habitual en la documentación de esta época, especialmente cuando la vocal tónica es una vocal cerrada /u/, como ocurre aquí (Morala, 2012b: 562). Para esta misma voz, aunque con otros valores, tenemos constancia de este fenómeno en el corpus: el adjetivo segura pasa a sigura en «la qual me obligo de os hazer sigura e de paz» (Medina Sidonia, Ca-1603) y el topónimo Segura sufre la misma alteración en «media tahulla de moreral junto al río Sigura» (Alcantarilla, Mu-1591) y en «la villa de Sigura de León» (Segura de León, Ba-1659).

29 Esta forma, segurón, se documenta igualmente en la documentación abulense de Mombeltrán en el siglo $\mathrm{xv}$ y, con anterioridad, en un área más amplia hacia el norte de la meseta (Sánchez González y Sánchez Romo, 2018: 312).

30 Esta referencia, que aparece también en el caso de segureja, nos indica que el uso de la segur no solo es equivalente a 'hacha para cortar leña, sino que cabe igualmente su utilización en el ámbito doméstico. 
discontinuidad geográfica con las áreas citadas: «una márcula con que se podan los olibos; una sigura y gabite» (Adeje, Tf-1695).

En cuanto a su registro en los atlas lingüísticos, segur o sus derivados cuentan con una presencia importante en varios de los territorios en los que los hemos localizado en los documentos, aunque con algunas diferencias. En el caso de Extremadura (CLEx, 102), es la voz más extendida para 'hacha' en la provincia de Cáceres, donde, más que la forma simple, se registran segurón, segurona, segureja, segurajilla o segureta. En Castilla y León ( $A L C y L, 416$ 'hacha'), las respuestas que implican este término (segurón, segureja, segurilla) se concentran casi en exclusiva en el sur de Salamanca y de Ávila, generalmente concurriendo con hacha y con destral y derivados, formando una evidente continuidad con el área de Cáceres citada. No hay, sin embargo, ejemplos ni en Segovia ni en Zamora o el noreste de León - como veíamos para el siglo XVII-, como tampoco en el resto de las dos provincias citadas ${ }^{31}$, en lo que debería analizarse como un evidente retroceso de segur frente al galicismo bacha.

Por lo que toca al área aragonesa — dominada por los resultados destral o estral-y en consonancia con la documentación aportada, el ALEANR (339 'hacha') presenta casos aislados de segur y su derivados tanto en Navarra como en Teruel (segur, segureta). Fuera de estas áreas, solo aparece algún caso aislado en otros mapas, como seguro o aseguro en puntos de Granada $(A L E A, 119)$ o seguro en un punto del norte de Palencia $(A L C y L, 391)$, en ambos casos para designar el calabozo.

\section{DESTRAL}

El DLE registra el término destral 'hacha pequeña', así como, a partir de la edición de 1925 $5^{32}$, una variante formal astral en Aragón, que en 2001 pasa a estral. Es voz bien representada en los repertorios lexicográficos, siempre como sustantivo masculino. La registra ya Nebrija, que la da como sinónimo de segur, mientras que Covarrubias apunta su origen en dextra, por la mano con la que se usa (NTLLE: $s$. v. destral). El DECH confirma esa etimología «del latino hispánico DEXTRALIs íd., así llamado porque se maneja con la mano derecha » e indica que «hoy se emplea estral o astral en el Alto Aragón ». Respecto a su extensión en castellano, J. Corominas y J. A. Pascual dan varios datos de interés para cruzarlos con nuestros documentos:

\footnotetext{
31 Únicamente rompe esta distribución un punto aislado en el ángulo nororiental de Salamanca (Sa-202) en el que se registran segurón y segurilla. s. v. estral).

32 Estral figura ya como provincialismo en el suplemento de Gaspar y Roig de 1853 (NTLLE:
} 
el término inicialmente significaría 'hacha pequeña que se coge con una sola mano', por oposición a segur, que se cogería con las dos; es voz que se extiende por todo el estrato antiguo del castellano oriental y se continúa en aragonés y catalán, donde se usa en femenino, género antiguo del que hoy sería muestra el diminutivo destraleja, pese a que la lexicografía española recoge destral siempre en masculino (DECH: $s$. v. diestro).

Nuestro corpus nos aporta un buen volumen de ejemplos, con algunas peculiaridades formales y un área de expansión que incluiría el aragonés y el castellano, principalmente en su área centro-oriental, sin ejemplos en Andalucía y solo alguno esporádico en Salamanca por lo que toca al área occidental ${ }^{33}$.

En el caso de los documentos aragoneses, el término aparece con regularidad en los inventarios procedentes de Huesca, mayoritariamente con la variante estral, además de un caso de estralilla, y, cuando el contexto permite identificarlo, usado en femenino:

una destral y un ajadón viejos (Ayerbe, Hu-1614); una estralilla pequeña; una estral sin mango (Plasencia del Monte, Hu-1614); vna estral grande (Villarreal de la Canal, Hu-1689); dos estrales, vna hoz de podar, seis hoces de segar (Loscertales, Hu-1653); dos destrales grandes (Monzón, Hu-1657); no hay destrales (Bielsa, Hu-1656).

Para el área oriental del castellano, contamos con ocurrencias en La Rioja, Soria, Cuenca - donde son más frecuentes los ejemplos-, Albacete y el occidente de Alicante, siempre con la forma destral y solo ocasionalmente en femenino (una destralica, una destral) al sur de dicha área:

un destral (Alfaro, LR-1647); un destral; otro destral (Soria, 1646); vn destral de haçer rajas (Cuenca, 1622); vn destral y una açuela (Villamayor de Santiago, Cu-1635); vna destralica (Almansa, Ab,1640); un destral pequeño (La Roda, Ab-1642); vn destral grande (Sax, A-1666); una destral (Sax, A-1639).

Por último, ha de señalarse otra área, en la zona del castellano central norteño, constituida básicamente por documentación de Cebreros (Ávila) y de Segovia, donde el término tiene una buena representación, así como algún punto de Toledo, aunque el documento es del siglo XVI. No aparece, sin embargo, en los documentos revisados de Burgos, Palencia o Valladolid. A esta área han de añadirse algunos ejem-

33 No hay, sin embargo, ejemplos en Asturias, León, Zamora o Extremadura. 
plos localizados en Ciudad Rodrigo (Salamanca). En Segovia se localiza el único caso del diminutivo lexicalizado destraleja, registrado ya por Nebrija como «destraleja o segureja»:

un destral ${ }^{34}$ con un pedaço de astil; una destraleja (Segovia, 1659); un destral $^{135}$ (Segovia, 1661); dos destrales de partir leña (Segovia, 1664); un destral y una hoz (Cebreros, Áv-1653); un destral (Cebreros, Áv-1654); vn destral (San Martín de Pusa, To-1532); vn destral (Ciudad Rodrigo, Sa-1630); vn destral (Ciudad Rodrigo, Sa-1633).

Esta distribución presenta algunas diferencias con los datos que nos ofrecen los atlas modernos. Por ejemplo, el $A L C y L$ (416), registra casos de destral prácticamente solo en Ávila y, sobre todo, en Salamanca, con algún punto en el oriente de Soria donde se recoge estral, pero no aparecen en el resto de Soria ni en Segovia, donde en el siglo XVII era frecuente y actualmente se ha impuesto hacha. Una situación similar a la que ocurre en La Rioja, mientras que estral y variantes son de uso general en buena parte de Aragón (ALEANR, 339). En el ALEA (716) la forma dominante es hacha, si bien hay algún punto aislado en el norte de Granada donde se registra destral. El ADiM (1164) da destralilla 'calabozo' en un punto del extremo suroccidental de la región (San Martín de Valdeiglesias), junto al límite con Ávila.

\section{GUADAN $A$}

La lexicografía española registra guadaña 'instrumento para segar' desde sus orígenes, sin mayor variación formal o semántica (NTLLE: s. v. guadaña). El término, de origen germánico (DECH: s. v. guadaña), es de uso general y así aparece también en nuestro corpus, representado en documentos que van de Cantabria a Jaén, pasando por León, Zamora, Burgos o Madrid.

una guadaña (Tanos, S-1672); dos aguadañas biejas (Treceño, S-1631); unos yerros de guadaña (Molinaferrera, Le-1698); vna guadaña con su vigornia y

\footnotetext{
34 Este ejemplo es interesante para ver cómo se superponen el patrimonial destral y el galicismo hacha. El documento es un inventario post mortem del que dos semanas después se hizo la correspondiente tasación, escrita ahora por una mano distinta y fechada en una localidad cercana a Segovia. Pues bien, en este segundo documento, lo que figura es hacha donde antes figuraba destral: «un acha buena mediana y otra más pequeña $\gg$ (Revenga, Sg-1659).

35 Unas líneas antes, en el mismo documento, se inventarían «dos siguras», lo que evidenciaría que segur y destral se usan con significados distintos en el texto.
} 
martillo (Abejera, Za-1689); vna tierra a do diçen tras la guadaña (Puentedura, Bu-1655); un guadaño (Torrelaguna, M-1657); vna guadaña para adereçar (Alcalá la Real, J-1648).

De los ejemplos de arriba, cabe destacar únicamente la variante en masculino (guadaño) documentada para Madrid - motivada seguramente por una diferencia de tamaño o de función - o el caso de aguadaña en Cantabria, por confusión con la /-a/ del artículo. Más interesante, desde el punto de vista formal, es constatar la preferencia por una forma con aféresis de la /u/ del diptongo (gadaña) en el área leonesa, forma que se sigue utilizando hoy - junto a gadañu o gadaño - y que es general también en gallego y portugués (DGLA: s. v. gadaña; Le Men: s. v. guadaña): «una gadaña con su junca y martillo» (Lumajo, Le-1646); «vna gadaña de segar yerba » (Brimeda, Le-1648); «vna gadaña bieja con su estil» (Mataluenga, Le-1671); «vna gadaña con su martillo y vigornia » (Villarino de Manzanas, Za-1683).

\section{DALLE}

El DLE registra tanto dalle 'guadaña' como dallar 'segar la hierba con el dalle'. En los repertorios lexicográficos figura por primera vez en Covarrubias, que lo define como 'arma', mientas que Autoridades lo hace como 'instrumento cortante, que regularmente sirve para cortar la yerba', definición que se mantiene en la lexicografía académica hasta la edición de 1884 , en la que pasa simplemente a 'guadaña' (NTLLE: s. v. dalle). A esta entrada se añade la variante dalla, que se incorpora en la edición de 1925 con el mismo significado y la marca de «en algunas comarcas», que, ya en 2001, se concreta en Aragón y Navarra (NTLLE: s. v. dalla).

J. Corominas y J. A. Pascual indican que el término está «tomado del cat. dall o de oc. dalh íd., procedentes de una voz DACŬLUM del latín tardío», pero advierten de que «este extranjerismo está escasamente documentado en castellano», mientras que dall y dalla (dalh, dalha) constituyen la denominación normal de la guadaña en catalán y en occitano. Como dalla sería forma aragonesa, consideran que dalla y dallar «pueden ser aragonesismos en castellano; pero dalle, con su -e, ha de proceder del catalán o de la lengua de $\mathrm{Oc} \gg(D E C H:$ s. v. dalle).

Los datos que aporta el CorLexIn son escasos, pero creo que tienen interés tanto por el ámbito geográfico que dibujan como por las variantes formales que se registran: «un dalle de segar yerba» (Soria, 1646); «un prado de dallo» (Padilla (Medinaceli), So-1675); «vna dalla con sus martillos» (Tordelrábano, Gu-1613); «una dalla con todos sus aderentes» (Tordelrábano, Gu-1616). 
En primer lugar, vemos que los testimonios de dalle en nuestro corpus se circunscriben al área limítrofe entre Guadalajara y Soria, sin que lo encontremos fuera de esta zona. Además, de los cuatro casos, tan solo uno figura con la forma académica (dalle), mientras que hay dos de dalla y uno de dallo ${ }^{36}$.

Si comparamos estos datos con los atlas lingüísticos (ALEANR, 509; ALECMan, 231), vemos que dalla, además de ser general en Aragón, se localiza en puntos del oriente de Soria, Cuenca y Albacete, así como en la mayor parte de Guadalaja$\mathrm{ra}^{37}$, en este caso alternando formas en /-o/ (el dallo $)^{38}$ - principalmente en el norte de la provincia - y formas en femenino (la dalla). Tanto por las formas como por la distribución geográfica, parece que estas voces han de considerarse un orientalismo, originado probablemente en el aragonés. Por el contrario, creo que es muy significativo que, mientras que en nuestro corpus no aparece ningún caso para Cantabria, el ALECant (373), registra de forma casi general, salvo en la zona occidental de Liébana, la forma dalle, con algunos puntos de dallo en el extremo oriental. El dato podría corroborar la expansión tardía del vocablo ${ }^{39}$, así como aportar información interesante para trazar los orígenes de la forma en /-e/ —un préstamo del catalán o del occitano según la teoría de Corominas - frente a las formas en /-a/, que habría que considerar patrimoniales en el castellano oriental.

\section{Conclusión}

Como hemos podido comprobar, el campo léxico elegido, el de las herramientas de corte, presenta en la lengua patrimonial - más concretamente, en los textos notariales del siglo XVII - una riqueza léxica sorprendente para lo esperable en un grupo de herramientas tan específicas. Especialmente si comparamos los datos del corpus utilizado con los de los atlas lingüísticos modernos, con los que presenta una evidente continuidad, frente a otras fuentes de información. No estamos hablando solo del registro documental de voces de uso más o menos local, sino también de la

36 La expresión prado de dallo sería equivalente a prado de guadaña, es decir, 'prado que se siega anualmente' $(D L E)$, por oposición a los que se explotan preferentemente para pasto.

37 En el norte de Madrid, lindando con Guadalajara, aparece un punto en el que se usan tanto guadaña como dalle, realizado como daye, con yeísmo, mientras que en el resto de la provincia todas las respuestas coinciden en guadaña (ADiM,1317).

38 Las variaciones de género se utilizan con frecuencia para marcar diferencias de significado. Hemos visto arriba algún caso de guadaño. En las escuetas enumeraciones de los notarios, es difícil definir cuáles son esas diferencias de significado.

39 Lo mismo puede decirse de varios puntos aislados de Burgos o Palencia en los que dalle aparece respectivamente con el sentido de 'calabozo' $(A L C y L, 391)$ y 'hoz' $(A L C y L, 259)$. 
posibilidad de identificar derivados que los corpus convencionales o lo diccionarios tienen dificultad para detectar, de localizar documentación antigua para voces que carecían de ella pese a ser de uso patrimonial, de identificar procesos de expansión o de reducción o, en fin, de fijar las isoglosas léxicas de voces que habitualmente se tienen por términos de uso no marcado diatópicamente. En último término, una muestra más de la complejidad del léxico histórico que, en el caso del léxico agrícola, presenta toda la variación propia de la lengua patrimonial y de las denominaciones localmente arraigadas.

\section{Bibliografía}

ADiM = García Mouton, Pilar e Isabel Molina Martos (2015): Atlas Dialectal de Madrid, Madrid, CSIC. En línea: <http://www.adim.cchs.csic.es> [30/06/2020].

ALCyL = Alvar, Manuel (1999): Atlas Lingüistico de Castilla y León (ALCyL). Junta de Castilla y León, Consejería de Educación y Cultura.

ALEA = Alvar, Manuel (1991): Atlas lingüistico y etnográfico de Andalucia, Madrid, Arco/Libros.

ALEANR = Alvar, Manuel, con la colaboración de Antonio Llorente MaLdonado de Guevara, Tomás Buesa Oliver y Elena Alvar (1981): Atlas lingüistico y etnográfico de Aragón, Navarra y La Rioja, Madrid, Institución «Fernando el Católico», Diputación de Zaragoza.

ALECant = Alvar, Manuel (1995): Atlas Lingüistico y Etnográfico de Cantabria, Madrid, Arco/Libros.

ALECMan = García Mouton, Pilar y Francisco Moreno Fernández (dirs.): Atlas Lingüistico (y etnográfico) de Castilla - La Mancha, Universidad de Alcalá. En línea: <http://www2.uah.es/alecman> [30/06/2020].

Alvar Ezquerra, Manuel (2000): Tesoro léxico de las hablas andaluzas, Madrid, Arco/Libros.

Ariza Viguera, Manuel (1995): «Leonesismos y occidentalismos en las lenguas y dialectos de España», Philologia hispalensis, 10, pp. 77-88.

$C D H=$ Instituto de Investigación Rafael Lapesa de la Real Academia Española (2013): Corpus del Nuevo diccionario histórico. En línea: <http:// web.frl.es/CNDHE/> [30/06/2020].

CLEx = GonzÁlez SAlgado, José Antonio: Cartografia lingüistica de Extremadura. En línea: <http://www.geolectos.com> [30/06/2020].

$C O R D E=$ Real ACADEmia Española: Banco de datos en línea Corpus diacróni- 
co del español. En línea <http://www.rae.es> [30/06/2020].

CorLexIn = Morala Rodríguez, José Ramón (dir.): Corpus Léxico de Inventarios. En línea: <http://web.frl.es/CORLEXIN.html> [30/06/2020].

$D E C=$ Corrales Zumbado, Cristóbal y Dolores Corbella Díaz (2009): Diccionario ejemplificado de canarismos, La Laguna, Instituto de Estudios Canarios.

$D E C H=$ Corominas, Joan y José Antonio Pascual (1980-1991): Diccionario Crítico Etimológico Castellano e Hispánico, 6 vols., Madrid, Gredos.

DGL $A=$ García Arias, Xosé Lluis (2015): Diccionario general de la lengua asturiana, Oviedo, Editorial Prensa Asturiana. En línea: <https://mas.lne.es/ diccionario/> [30/06/2020].

DHCan = Corrales Zumbado, Cristóbal y Dolores Corbella Díaz: Diccionario Histórico del Español de Canarias. En línea: <http://web.frl.es/DHECan.html> [30/06/2020].

DLE $=$ Real Academia Española: Diccionario de la Lengua Española. En línea: <http://www.rae.es> [30/06/2020].

Gómez Ferrero, Cristina (2015): Estudio del léxico en los protocolos notariales del partido judicial de La Bañeza (ss. XVII-XIX), Tesis doctoral inédita, Universidad de León. En línea: <https://dialnet.unirioja.es/servlet/tesis?codigo=44909> [30/06/2020].

González Casarrubios, Consolación y María Elisa Sánchez Sanz (1978): «El Rabel», Narria: Estudios de artes y costumbres populares, 9, pp. 21-25.

Le Men Loyer, Janick (2002-2009): Léxico del leonés actual, León, Centro de Estudios e Investigación «San Isidoro».

Morala Rodríguez, José Ramón (2008): «Léxico en cartas de examen del siglo XVII », en A. Álvarez et alii (eds.), Lengua viva. Estudios ofrecidos a César Hernández Alonso, Valladolid, Universidad de Valladolid/Diputación de Valladolid, pp. 575-590.

Morala Rodríguez, José Ramón (2012a): «El proyecto CorLexIn», en Dolores Corbella, Josefa Dorta, Alejandro Fajardo Aguirre, Laura Izquierdo, Javier Medina López y Antonia Nelsi Torres (eds.), Lexicografía hispánica del siglo XXI: nuevos proyectos y perspectivas. Homenaje al Profesor Cristóbal Corrales Zumbado, Madrid, Arco/Libros, pp. 421-439.

Morala Rodríguez, José Ramón (2012b): «Alternancias en el vocalismo átono en textos notariales del siglo XVII », en E. Montero Cartelle (ed.), Actas del VIII Congreso Internacional de Historia de la Lengua Española, Santiago de Compostela, Meubook, vol. I, pp. 555-564.

Morala Rodríguez, José Ramón (2017a): «Voces derivadas en documentación 
notarial del siglo XvII», Cuadernos del Instituto de Historia de la Lengua, 10, pp. 135-163.

Morala Rodríguez, José Ramón (2017b): «Algunos derivados en -il en un corpus del siglo XVII», en Florencio del Barrio de la Rosa (ed.), Palabras Vocabulario Léxico. La lexicología aplicada a la didáctica y a la diacronia, Venezia, Edizioni Ca' Foscari, pp. 267-281.

NTLLE = Real ACAdemia Española: Nuevo Tesoro Lexicográfico de la Lengua Española. En línea: <http://www.rae.es> [30/06/2020].

Sánchez González de Herrero, M.a Nieves y Raquel Sánchez Romo (2018): «Sobre léxico de la documentación notarial medieval del sur de Ávila: léxico de la vida cotidiana», en Mónica Castillo Lluch y Elena Diez del Corral Areta (eds.), Reescribiendo la historia de la lengua española a partir de la edición de documentos, Bern, Peter Lang, pp. 293-317.

Fecha de recepción: 30 de junio de 2020 Fecha de aceptación: 3 de septiembre de 2020 